\title{
AVIAN INFLUENZA ('BIRD FLU')
}

\section{WHAT IS AVIAN INFLUENZA (BIRD FLU)?}

Avian influenza is an infectious disease of birds, caused by a number of different strains of avian influenza virus. Usually the virus circulates in wild bird populations causing no disease or only mild disease. Infection of domestic poultry, such as chickens, can cause severe disease in these birds. There are a number of different strains of avian influenza, only a few of which can cause disease in humans.

A new type of avian influenza, called influenza A H5N1 (hereafter referred to as H5N1), was first recognised in 1997 in Hong Kong. This strain reappeared in late 2003 and has rapidly spread to many Asian, Middle-Eastern, European and African countries, causing severe infection in wild birds and domestic poultry flocks. There is no evidence that avian influenza is currently infecting birds in Australia.

This virus has also infected a number of people in Asia, the Middle East and Africa who had close contact with infected poultry or poultry droppings.

At this stage, humans infected with the H5N1 virus do not easily transmit the infection to others. Exposure to infected poultry, or their infected secretions or droppings, or dust or soil contaminated with their secretions or droppings, can result in human infection. Eating cooked poultry products including chicken or eggs does not result in infection.

A large-scale, worldwide influenza epidemic is called a pandemic. Pandemics occur when a new virus emerges to which people have little or no immunity. Previous influenza pandemics occurred in 1918-19, 1957-58 and 1968-69. In the 1918-19 pandemic, between 20 and 40 million people died. Many scientists are concerned that the recent H5N1 outbreak in birds could mutate to produce a new strain of influenza virus that is easily spread among people, resulting in a pandemic.

\section{WHAT ARE THE SYMPTOMS?}

Different strains of avian influenza can lead to different symptoms in people. All strains can cause symptoms typical of human influenza (fever, cough, tiredness, muscle aches, sore throat, shortness of breath, runny nose, headache). In some cases the H5N1 strain has caused a severe pneumonia and, in a small number of cases, encephalitis (inflammation of the brain) or diarrhoea. The most common symptom of humans infected with $\mathrm{H} 7$ strains of avian influenza is conjunctivitis (inflammation of the lining of the eye). Symptoms generally appear between two to four days following exposure.

\section{WHO IS AT RISK?}

Most people are not at risk of this disease. People at risk of becoming infected with $\mathrm{H} 5 \mathrm{~N} 1$ are those who come into contact with sick birds or their secretions or droppings while

\section{NSW@HEALTH}

living or travelling in areas where the virus is circulating, or (possibly) people who have had close contact with a person with the human form of the disease in the affected areas. In the absence of a vaccine, the best method of prevention is to ensure that all people who are working with infected birds are supplied with appropriate personal protective equipment, such as masks, goggles, gloves and protective clothing, and, if necessary, anti-influenza medication.

\section{HOW IS IT PREVENTED?}

A human vaccine is not available for the new avian influenza strain. Existing vaccines for normal human influenza will not provide protection against avian influenza, including the H5N1 strain. Scientists worldwide are currently working to develop a suitable vaccine for this strain.

\section{HOW IS IT DIAGNOSED?}

Avian influenza virus infection can be diagnosed using specimens of blood, or from swabs of the nose and throat. Testing is done at a specialised laboratory.

\section{HOW IS IT TREATED?}

Specific anti-influenza drugs are likely to be effective against avian influenza in humans and are used to treat people with the H5N1 strain.

\section{WHAT IS THE PUBLIC HEALTH RESPONSE?}

Outbreaks of different strains of avian influenza have occurred previously in Australia. However, there have been no recent reports of avian influenza in Australian birds and there are no reports of Australian people with H5N1 virus infection. There is surveillance for the illegal importation of birds or bird products at Australian borders.

Human infection with avian influenza must be notified to the local public health unit. Should suspected human cases occur in NSW, the local public health unit would work with the patient, the treating doctors, and the laboratory to confirm the diagnosis. Suspected cases would be isolated from others to prevent further infections. Close contacts of these cases who may have been exposed to the virus will be given information about the risk of infection. Should these people also develop symptoms, they would also be isolated and tested for avian influenza.

\section{TRAVEL ADVICE}

Australians travelling to areas affected by avian influenza should reduce their risk of infection by avoiding poultry farms and live bird markets. They should also ensure that they wash their hands thoroughly after handling uncooked poultry products such as eggs, and that they ensure that poultry is cooked thoroughly before eating.

For more information contact the Australian Government Department of Health and Ageing information hotline 1800004599 . 종 